\title{
Rydberg-state mixing in the presence of an external electric field: Comparison of the hydrogen and antihydrogen spectra
}

\author{
D. Solovyev ${ }^{1, *}$ and E. Solovyeva ${ }^{2}$ \\ ${ }^{1}$ Department of Physics, Saint Petersburg State University, Petrodvorets, Oulianovskaya 1, 198504 Saint Petersburg, Russia \\ ${ }^{2}$ Department of Chemistry, Saint Petersburg State University, Petrodvoretz, Universitetsky Prospect 26, 198504 Saint Petersburg, Russia
}

(Received 30 December 2014; published 16 April 2015)

\begin{abstract}
We analyze the influence of an external electric field on the $n s$ and $n p$ Rydberg states. The impact of an electric field leads to a significant modification of the decay rate. Namely, the additional (linear and quadratic in the field) terms arise in the differential transition probabilities. In contrast to the quadratic term, the linear summand leads to a difference of the hydrogen $(\mathrm{H})$ and antihydrogen $(\overline{\mathrm{H}})$ spectra. However, this difference vanishes after the integration over photon-emission directions and does not contribute to the total emission probability. The quadratic term results in the significant change of the total decay rate and level width, respectively. The analysis of the linear and quadratic terms depending on the field strength is discussed.
\end{abstract}

DOI: 10.1103/PhysRevA.91.042506

PACS number(s): 32.30.-r, 32.70.Jz, 32.80.Ee, 32.70.Cs

\section{INTRODUCTION}

One of the most fundamental problems of modern physics is to test the charge-parity-time $(\mathcal{C P} \mathcal{T})$ symmetry and the search for the effect of its violation. In principle, the detailed comparison of the hydrogen $(\mathrm{H})$ and antihydrogen $(\overline{\mathrm{H}})$ spectra can be used for this purposes. In fact, the hydrogen atom is currently the most studied quantum system. Very precise experiments correspond to the measurements of the spectroscopic properties of this atom. In particular, the frequency measurements of the two-photon transitions $2 s-1 s$ [1-3] and $3 s-1 s$ or $3 d-1 s[4,5]$ in hydrogen have established the highest order of accuracy for the spectroscopic experiments. On the other hand, the recent success in the production of antihydrogen atoms offers the possibility for a detailed comparison of spectroscopic properties of the $\mathrm{H}$ and $\overline{\mathrm{H}}$ atoms. As it was reported in [6], the antihydrogen atoms were confined in a trap for about $1000 \mathrm{~s}$. Such a time of trapping allows, in principle, the spectroscopic experiments with the antihydrogen atoms.

In accord with the $\mathcal{C P} \mathcal{T}$ theorem, the spectra of matter and antimatter atoms should be identical. Nonetheless, it was shown that signals for $\mathcal{C P} \mathcal{T}$ symmetry and the Lorentz violation at the Planck scale may arise in hydrogen and antihydrogen spectroscopy [7]. Effects of this type can appear in $\mathrm{H}$ and $\overline{\mathrm{H}}$ spectra at zeroth order in the fine-structure constant and are detectable not only in spin-mixed $1 s-2 s$ lines but also in spin-flip hyperfine signals. Moreover, superaccurate measurements of the transition frequency in hydrogen have stimulated the theoretical studies of some exotic quantum corrections [8]. Such corrections reveal a specific difference in $\mathrm{H}$ and $\overline{\mathrm{H}}$ atomic spectra in the presence of the external electric field. However, this difference is very small and hence the existence of the electric stray field should not become a serious problem in experiments searching for $\mathcal{C P} \mathcal{T}$-violating effects.

Recently, spectra of the hydrogen and antihydrogen atoms were compared in the case of the presence of an external electric field $[9,10]$, where the one- and two-photon transition

*solovyev.d@gmail.com rates for the decays of mixed $\overline{2 s}$ and $\overline{2 p}$ states were evaluated. It was shown that the term linear in the field arising in the differential transition probability leads to a significant difference of the $\mathrm{H}$ and $\overline{\mathrm{H}}$ spectra. This deviation vanishes after integration over photon-emission directions, i.e., in the total transition probability. Nonetheless, the difference arising in the electric field can be observed in experiments when the photon emission is registered in a predetermined direction. According to [6], production of antihydrogen atoms in the low-lying states is difficult at present and thus a theoretical analysis of the decays of highly excited states is needed.

Employing the technique of $[9,10]$, in this paper we evaluate the transition probabilities for the decays of highly excited states. In particular, in Sec. II we describe the mixing effect arising in an external electric field for the highly excited atomic levels. In Sec. III the comparison of differential transition probabilities for the $\mathrm{H}$ and $\overline{\mathrm{H}}$ atoms is illustrated using the example of $n s$ and $n p$ states mixing. In Sec. IV the total decay rates (identical for the $\mathrm{H}$ and $\overline{\mathrm{H}}$ atoms) of mixed $\overline{n s}$ states are calculated for arbitrary principal quantum numbers of the initial and final states. The choice of $n s$ and $n p$ states is due mainly by the fact that the mixing is stronger for close states of opposite parity. In this case, the Lamb shift acts as a parameter, allowing $n s$ and $n p$ states to be fully mixed in very weak fields, which can be associated with stray fields. A discussion of the results and a summary are given in Sec. V. The main goal of this paper is to demonstrate the difference in the spectra of hydrogen and antihydrogen atoms for Rydberg states, which can mimic the effects of $\mathcal{C P} \mathcal{T}$ violation.

\section{RYDBERG-STATE MIXING}

As it was shown in $[9,10]$, the mixing of $2 s$ and $2 p$ states by an external electric field in the hydrogen atom can lead to an essential modification of the transition probabilities. This modification is determined by the terms linear and quadratic in the field. The linear term has a special meaning in the analysis of $\mathrm{H}$ and $\overline{\mathrm{H}}$ spectra. Namely, the linear part of the transition probability has the opposite sign (due to the opposite sign of the charge) for these atoms, thereby creating a difference in the corresponding decay rates. In turn, the term quadratic in 
the field contributes to the corresponding level width but is the same for the $\mathrm{H}$ and $\overline{\mathrm{H}}$ atoms. Below we apply the technique developed in $[9,10]$ and consider the influence of an external electric field on the decay rates of $n s$ Rydberg states.

According to the perturbation theory, the wave function of the atomic level subjected to the action of an external electric field can be expressed as [11,12]

$$
\begin{aligned}
\left|\overline{n s} m_{j_{s}}\right\rangle & =\left|n s m_{j_{s}}\right\rangle+\eta_{n} \sum_{m_{j_{p}}}\left\langle n p m_{j_{p}}|e \mathbf{D r}| n s m_{j_{s}}\right\rangle\left|n p m_{j_{p}}\right\rangle \\
\eta_{n} & =\frac{1}{\Delta E_{L(f)}^{(n)}+i \Gamma_{n p} / 2}
\end{aligned}
$$

where $m_{j_{s(p)}}$ corresponds to the projection of the total angular momentum $j_{s(p)}$ of the $n s(p)$ state, $n$ is the principal quantum number of the hydrogenic state, $\Delta E_{L(f)}^{(n)}$ is the Lamb shift ( $L$ index) or the fine-structure splitting $f$ of the $n$th atomic state, $\Gamma_{n p}$ is the width of the $n p$ level, and $e$ is the electron charge. The matrix element $\left\langle n p m_{j_{p}}|e \mathbf{D r}| n s m_{j_{s}}\right\rangle$ represents the dipole interaction of the atomic electron with the external-electricfield vector $\mathbf{D}$ and $\mathbf{r}$ is the radius vector of the atomic electron.

In principle, the mixing of states with the principal quantum numbers $n$ and $n \pm 1$ should be taken into account also. However, it is easy to show analytically that the corresponding matrix element in Eq. (1) leads to a correction smaller than the main contribution due to the Lamb shift since $\Gamma_{n p} \ll \Delta E_{L}^{(n)} \ll$ $\Delta E_{f}^{(n)} \ll \Delta E_{n, n \pm 1}$, where $\Delta E_{n, n \pm 1}$ is the energy difference for the $n$th and $n \pm 1$ th states. After integration over angular and radial variables in Eq. (1) we arrive at

$$
\begin{aligned}
& \left\langle n m_{j_{p}}|e \mathbf{D r}| n s m_{j_{s}}\right\rangle \\
& \quad=\frac{\sqrt{3}}{2} n \sqrt{n^{2}-1} \sum_{q}(-1)^{q+j_{s}+j_{p}} e D_{q} C_{j_{s} m_{j_{s}} 1-q}^{j_{j_{j} m_{j_{p}}}} .
\end{aligned}
$$

Here $C_{j_{1} m_{1} j_{2} m_{2}}^{j m}$ is the Clebsch-Gordan coefficient with the total angular momentum $j$ and its projection $m$ defined via the rule of vector summation for the angular moments $j_{1}$ and $j_{2}$ and their projections $m_{1}$ and $m_{2}$, respectively, and $D_{q}$ is the radial component $q$ of the electric-field vector $\mathbf{D}$.
The amplitude of the one-photon transition in the Pauli approximation can be written in the form [10]

$$
U_{A^{\prime} A}^{P}(\mathbf{k}, \mathbf{e})=\left\{(\mathbf{e} \hat{\mathbf{p}}+i \mathbf{e}[\mathbf{k} \times \mathbf{s}]) e^{-i \mathbf{k r}}\right\}_{A^{\prime} A},
$$

where the second term corresponds to the Pauli approximation, the operator $\hat{\mathbf{p}}$ represents the momentum of the atomic electron, $\mathbf{e}$ is the photon polarization vector, $\mathbf{k}$ is the photon wave vector with frequency $\omega(|\mathbf{k}| \equiv \omega)$, s represents the spin operator, and $A^{\prime}$ and $A$ are the final and initial states, respectively, given by the solution of the Schrödinger equation. Then the one-photon transition probability is

$$
W_{A^{\prime} A}^{(1 \gamma)}=\frac{e^{2} \omega_{A A^{\prime}}}{2 \pi} \sum_{\mathbf{e}} \int d \mathbf{n}_{\mathbf{k}}\left|U_{A^{\prime} A}^{P}(\mathbf{k}, \mathbf{e})\right|^{2},
$$

where $\mathbf{n}_{\mathbf{k}} \equiv \mathbf{k} / \omega$ is the unit vector in the direction of the photon emission and $\omega_{A^{\prime} A}$ is the transition frequency $\omega_{A^{\prime} A}=E_{A}-$ $E_{A^{\prime}}$ with the energies of the atomic electron $E_{A}$ and $E_{A^{\prime}}$ for states $A$ and $A^{\prime}$, respectively.

Consider now the one-photon transition in the presence of an external electric field. According to the selection rules, the term corresponding to the unperturbed initial $n s$ state in Eq. (1) gives the emission of the magnetic dipole $M 1$ photon. In turn, the term dependent on the electric-field strength $\mathbf{D}$ in Eq. (1) leads to the electric dipole $E 1$ emission [the $n p \rightarrow$ $k s+1 \gamma(E 1)$ decay channel]. For the complete evaluation of the decay rate, the mixing of low-lying states should be taken into account also, i.e., the $n s \rightarrow k p+1 \gamma(E 1)$ decay channels. Therefore, the total amplitude of the emission process $\overline{n s} \rightarrow$ $\overline{k s}+1 \gamma$ can be written as

$$
\begin{aligned}
U_{\overline{n s} m, \overline{k s} m^{\prime}}^{P}(\mathbf{k}, \mathbf{e})= & U_{n s m, k s m^{\prime}}^{P}(\mathbf{k}, \mathbf{e}) \\
& +\eta_{n} \sum_{m_{j_{p}}}\left\langle n s m|e \mathbf{D r}| n p m_{j_{p}}\right\rangle U_{n p m_{j_{p}}, k s m^{\prime}}^{P}(\mathbf{k}, \mathbf{e}) \\
& +\eta_{k} \sum_{m_{j_{p}}}\left\langle k p m_{j_{p}}|e \mathbf{D r}| k s m^{\prime}\right\rangle U_{n s m, k p m_{j_{p}}}^{P}(\mathbf{k}, \mathbf{e}),
\end{aligned}
$$

where projections $m$ and $m^{\prime}$ correspond to the total angular moments of the initial $n s$ and final $k s$ states, respectively. To derive the transition probability we use Eq. (5) in conjunction with summing over polarization e and projection $m^{\prime}$ and averaging over projection of the initial state $m$. Therefore,

$$
\begin{aligned}
d W_{\bar{n} s k s}^{(1 \gamma)}\left(\mathbf{n}_{\mathbf{k}}\right)= & \frac{3}{8 \pi} d W_{n s k s}^{(1 \gamma)}\left(\mathbf{n}_{\mathbf{k}}\right)\left[1-(-1)^{j_{s}+j_{p}} e D_{0}\left(\mathbf{n}_{\mathbf{D}} \mathbf{n}_{\mathbf{k}}\right) \frac{n \sqrt{n^{2}-1}}{2 \sqrt{3}} \frac{\Gamma_{n p}}{w_{1} \Delta_{1}^{2}}-(-1)^{j_{s}+j_{p}} e D_{0}\left(\mathbf{n}_{\mathbf{D}} \mathbf{n}_{\mathbf{k}}\right) \frac{k \sqrt{k^{2}-1}}{6} \frac{\Gamma_{k p}}{w_{2} \Delta_{2}^{2}}\right. \\
& \left.+\frac{e^{2} D_{0}^{2}}{w_{1}^{2} \Delta_{1}^{2}} \frac{n^{2}\left(n^{2}-1\right)}{12}+\frac{e^{2} D_{0}^{2}}{w_{2}^{2} \Delta_{2}^{2}} \frac{k^{2}\left(k^{2}-1\right)}{36}\right] d \mathbf{n}_{\mathbf{k}} .
\end{aligned}
$$

Here $\mathbf{n}_{\mathbf{D}}$ is the unit vector in the field direction $\left(\mathbf{n}_{\mathbf{D}} \equiv \mathbf{D} / D_{0}\right.$, where $D_{0}$ is the field amplitude) and the parameters $\Delta_{i}$ and $w_{i}$ $(i=1,2)$ are defined as follows:

$$
\Delta_{1}=\sqrt{\left(\Delta E_{L(f)}^{(n)}\right)^{2}+\frac{1}{4} \Gamma_{n p}^{2}}, \quad \Delta_{2}=\sqrt{\left(\Delta E_{L(f)}^{(k)}\right)^{2}+\frac{1}{4} \Gamma_{k p}^{2}}, \quad w_{1}=\sqrt{\frac{W_{n s k s}^{(1 \gamma)}}{W_{n p k s}^{(1 \gamma)}}}, \quad w_{2}=\sqrt{\frac{W_{n s k s}^{(1 \gamma)}}{W_{n s k p}^{(1 \gamma)}}} .
$$


Here we suppose that the width of the $n p$ state $\Gamma_{n p}=\Gamma_{n p_{1 / 2}}=$ $\Gamma_{n p_{3 / 2}}$ [13]. The notation $W_{n s k s}^{(1 \gamma)}, W_{n p k s}^{(1 \gamma)}$, and $W_{n s k p}^{(1 \gamma)}$ represents the one-photon transition probabilities for the corresponding $n s$ and $n p$ levels. The sign $(-1)^{j_{s}+j_{p}}$ in Eq. (6) reveals the opposite contribution of the $n p_{1 / 2}$ and $n p_{3 / 2}$ states. This difference arises because the $n s$ state is located between $n p_{3 / 2}$ and $n p_{1 / 2}$.

In expression (6) the last two terms do not depend on the photon emission or the electric-field directions. In contrast to the contribution of linear terms, the summand quadratic in a field [last line in Eq. (6)] survives after the averaging over field directions or after the integration over photon-emission directions. We should note that the formal $T$ noninvariance of the factor $\mathbf{n}_{\mathbf{D}} \mathbf{n}_{\mathbf{k}}$ in Eq. (6) ( $T$-odd and $T$-even vectors, respectively) is compensated by the dependence on $\Gamma_{n(k) p}$ in the numerator on the right-hand side of Eq. (6) [14].

\section{HYDROGEN AND ANTIHYDROGEN SPECTROSCOPY}

According to Eq. (6), the differential transition probability $d W_{\overline{n s} \overline{k s}}^{(1 \gamma)}\left(\mathbf{n}_{\mathbf{k}}\right)$ is constructed as follows: The first term corresponds to the unperturbed $n s \rightarrow k s+1 \gamma(M 1)$ decay and the additional terms (linear and quadratic in the electric field $D_{0}$ ) represent the interference of the $M 1$ and $E 1$ photon emissions and the decay rates $n s \rightarrow k p+1 \gamma(E 1)$ and $n p \rightarrow k s+1 \gamma(E 1)$. The linear terms are proportional to the electron charge, therefore they have the opposite sign for the $\mathrm{H}$ and $\overline{\mathrm{H}}$ atoms. All the other summands in Eq. (6) are identical for the hydrogen and antihydrogen. Note that the linear dependence on the field vanishes in the total decay rate, i.e., after integration over photon-emission directions. The observation of the contrast in hydrogen and antihydrogen spectra is sharper when the photon emission is detected at the angle in the forward or backward field direction.

For the comparison of the differential transition probabilities it is more convenient to present Eq. (6) in the form

$$
\begin{aligned}
d W_{\overline{n s} k s}^{(1 \gamma)}= & \frac{3}{8 \pi} d W_{n s k s}^{(1 \gamma)}\left(1+e^{2} D_{0}^{2} a^{2}\right) \\
& \times\left[1 \pm(-1)^{1+j_{s}+j_{p}} \frac{e D_{0} b\left(\mathbf{n}_{\mathbf{D}} \mathbf{n}_{\mathbf{k}}\right)}{1+e^{2} D_{0}^{2} a^{2}}\right]
\end{aligned}
$$

where the signs \pm in Eq. (8) correspond to the hydrogen $(+)$ and antihydrogen $(-)$ atoms and the electron charge $e$ is included as the modulus. The coefficients $a$ and $b$ are defined as

$$
\begin{aligned}
& a=\sqrt{\frac{n^{2}\left(n^{2}-1\right)}{12 w_{1}^{2} \Delta_{1}^{2}}+\frac{k^{2}\left(k^{2}-1\right)}{36 w_{2}^{2} \Delta_{2}^{2}}}, \\
& b=\frac{n \sqrt{n^{2}-1}}{2 \sqrt{3}} \frac{\Gamma_{n p}}{w_{1} \Delta_{1}^{2}}+\frac{k \sqrt{k^{2}-1}}{6} \frac{\Gamma_{k p}}{w_{2} \Delta_{2}^{2}} .
\end{aligned}
$$

The second term in square brackets in Eq. (8) represents the expected difference in spectra of hydrogen and antihydrogen atoms in an external electric field. The maximum of this term can be found from the extremum condition that gives

$$
e D_{0}^{\max }=\frac{1}{a}
$$

The relative difference between the transition probabilities $d W_{\overline{n s} \overline{k s}}^{(1 \gamma)}(H)$ and $d W_{\overline{n s}}^{(1 \gamma)}(\bar{H})$ for $\mathrm{H}$ and $\overline{\mathrm{H}}$ atoms reaches a
TABLE I. Numerical values of the $\delta\left(D_{0}\right)$ for different values of the principal quantum numbers of the initial $n$ and final $k$ states. The third column represents the magnitude of $D_{0}^{\max }$ in $\mathrm{V} / \mathrm{m}$ depending on the principal quantum numbers $n$ and $k$. In the fourth and fifth columns the values of $\delta\left(D_{0}^{\max }\right)$ and $\delta\left(D_{0}\right)$ at $D_{0}=500 \mathrm{~V} / \mathrm{m}$ are given in the case of $\mathbf{n}_{\mathbf{D}} \mathbf{n}_{\mathbf{k}}=1$, i.e., when the detection of a photon occurs in the direction of the field.

\begin{tabular}{lcccc}
\hline \hline$n$ & $k$ & $D_{0}^{\max }$ & $\delta\left(D_{0}^{\max }\right)$ & $\delta\left(D_{0}\right)$ \\
\hline 2 & 1 & 0.005 & 0.094 & $1.97 \times 10^{-6}$ \\
3 & 1 & 0.0009 & 0.087 & $3.14 \times 10^{-7}$ \\
3 & 2 & 0.0001 & 0.094 & $3.77 \times 10^{-8}$ \\
4 & 1 & 0.0002 & 0.097 & $7.94 \times 10^{-8}$ \\
4 & 2 & 0.00003 & 0.098 & $1.18 \times 10^{-7}$ \\
4 & 3 & $5.9 \times 10^{-6}$ & 0.11 & $2.60 \times 10^{-9}$ \\
100 & 1 & $1.6 \times 10^{-11}$ & 0.099 & $6.33 \times 10^{-15}$ \\
100 & 2 & $4.2 \times 10^{-11}$ & 0.099 & $1.65 \times 10^{-14}$ \\
100 & 3 & $7.2 \times 10^{-11}$ & 0.099 & $2.85 \times 10^{-14}$ \\
\hline \hline
\end{tabular}

maximum in the field equation (10) and is equal to

$$
\begin{aligned}
\delta( & \left.D_{0}^{\max }\right) \\
= & \frac{d W_{\overline{n s} k s}^{(1 \gamma)}(H)-d W_{\overline{n s} \overline{k s}}^{(1 \gamma)}(\bar{H})}{(3 / 8 \pi) W_{n s k s}^{(1 \gamma)}\left(1+e^{2} D_{0}^{2} a\right)} \\
= & (-1)^{1+j_{s}+j_{p}}\left(\mathbf{n}_{\mathbf{D}} \mathbf{n}_{\mathbf{k}}\right) \frac{b}{a} \\
= & (-1)^{1+j_{s}+j_{p}}\left(\mathbf{n}_{\mathbf{D}} \mathbf{n}_{\mathbf{k}}\right) \\
& \times \frac{n \sqrt{n^{2}-1} \Gamma_{n p} / 2 \sqrt{3} w_{1} \Delta_{1}^{2}+k \sqrt{k^{2}-1} \Gamma_{k p} / 6 w_{2} \Delta_{2}^{2}}{\sqrt{n^{2}\left(n^{2}-1\right) / 12 w_{1}^{2} \Delta_{1}^{2}+k^{2}\left(k^{2}-1\right) / 36 w_{2}^{2} \Delta_{2}^{2}}} .
\end{aligned}
$$

The case when $n=2$ and $k=1$ (the case of $2 s-2 p$ mixing) leads to the result [10]. We should note that the admixture of $n p_{1 / 2}$ and $n p_{3 / 2}$ states contributes to $\delta\left(D_{0}^{\max }\right)$ with opposite sign. However, the admixture of the $n p_{3 / 2}$ state is smaller since $\Delta E_{L}^{(n)} \ll \Delta E_{f}^{(n)}$. Thus we can neglect the fine-structure splitting with respect to the Lamb shift. Moreover, expression (11) can be simplified significantly by applying the series expansion in powers of $1 / n$. The dominant contribution in both cases $k \ll n$ and $k \sim n$ is

$$
\delta\left(D_{0}^{\max }\right) \approx\left(\mathbf{n}_{\mathbf{D}} \mathbf{n}_{\mathbf{k}}\right) \frac{\Gamma_{n p}}{\Delta_{1}} \approx\left(\mathbf{n}_{\mathbf{D}} \mathbf{n}_{\mathbf{k}}\right) \frac{\Gamma_{n p}}{\Delta E_{L}^{(n)}}
$$

with the condition $\Gamma_{n p} \ll E_{L}^{(n)}$.

The results (11) and (12) allow the determination of spectral difference between $\mathrm{H}$ and $\overline{\mathrm{H}}$ atoms depending on the principal quantum numbers $n$ and $k$ of the initial and final states, respectively. In Table I we give the values of the dimensionless function $\delta\left(D_{0}\right)$ [Eq. (11)] depending on the values of the principal quantum numbers $n$ and $k$ for the electric-field magnitude $D_{0}^{\max }$ [Eq. (10)] and $500 \mathrm{~V} / \mathrm{m}$. In the last column of Table I, the electric-field strength is associated with the experimental value. We should note that the $D_{0}^{\max }$ in Table I represent rather the magnitudes that can be referred to a stray fields. 
TABLE II. Numerical values of $W_{1} \equiv \frac{n^{2}\left(n^{2}-1\right)}{12} e^{2} D_{0}^{2} W_{n p k s}^{(1 \gamma)} / \Delta^{2}$ and $W_{2} \equiv \frac{k^{2}\left(k^{2}-1\right)}{36} \frac{e^{2} D_{0}^{2} W_{n s k p}^{(1 \gamma)}}{\Delta_{2}^{2}}$ for different values of the principal quantum numbers of the initial $n$ and final $k$ states presented in inverse seconds. In the first and second columns the values of the principal quantum numbers $n$ and $k$ are listed. The third column gives the values of transition probabilities $W_{n s k s}^{(1 \gamma)}$ and the values of $W_{n p k s}^{(1 \gamma)}$ in s ${ }^{-1}$ are given in the fourth column [21-23]. The fifth column gives the $W_{n p k s}^{(1 \gamma)}$ transition probabilities in s ${ }^{-1}$. The used values of the Lamb shift $\Delta E_{L}$ in $M H z$ are given in the sixth column. In the seventh and eighth columns the values of the natural width of levels $\Gamma_{n p}$ and $\Gamma_{n s}$, respectively, are listed in $\mathrm{s}^{-1}$. The $\Gamma_{n p}$ and $\Gamma_{n s}$ are obtained as the sum of all partial $E 1$ transition probabilities to the low-lying states. The ninth and tenth columns give the contributions of the quadratic terms (13). The field strength $D_{c}^{(n)}$ was used, which approximately causes the full mixing of corresponding states.

\begin{tabular}{|c|c|c|c|c|c|c|c|c|c|}
\hline$n$ & $k$ & $W_{n s k s}^{(1 \gamma)}$ & $W_{n s k p}^{(1 \gamma)}$ & $W_{n p k s}^{(1 \gamma)}$ & $\Delta E_{L}^{(n)}$ & $\Gamma_{n p}$ & $\Gamma_{n s}$ & $W_{1}$ & $W_{2}$ \\
\hline 2 & 1 & $2.495 \times 10^{-6}$ & & $6.265 \times 10^{8}$ & 1057.911 & $6.265 \times 10^{8}$ & 8.229 & $2.063 \times 10^{8}$ & 0 \\
\hline 3 & 1 & $1.109 \times 10^{-6}$ & & $1.672 \times 10^{8}$ & 344.896 & $1.897 \times 10^{8}$ & $6.314 \times 10^{6}$ & $5.394 \times 10^{6}$ & 0 \\
\hline 3 & 2 & $1.877 \times 10^{-9}$ & $6.314 \times 10^{6}$ & $2.245 \times 10^{7}$ & 344.896 & & & $7.2398 \times 10^{6}$ & $1.2021 \times 10^{5}$ \\
\hline 4 & 1 & $5.303 \times 10^{-7}$ & & $6.819 \times 10^{7}$ & 133.084 & $8.092 \times 10^{7}$ & $4.414 \times 10^{6}$ & $2.771 \times 10^{7}$ & 0 \\
\hline 4 & 2 & $1.617 \times 10^{-9}$ & $2.578 \times 10^{6}$ & $9.668 \times 10^{6}$ & 133.084 & & & $3.929 \times 10^{6}$ & 276.37 \\
\hline 4 & 3 & $2.047 \times 10^{-11}$ & $1.836 \times 10^{6}$ & $3.065 \times 10^{6}$ & 133.084 & & & $1.246 \times 10^{6}$ & $1.111 \times 10^{4}$ \\
\hline 100 & 1 & $3.949 \times 10^{-11}$ & & $4.185 \times 10^{3}$ & $1.058 \times 10^{-3}$ & $\approx 5.25 \times 10^{3}$ & $\approx 0.476 \times 10^{3}$ & $6.371 \times 10^{3}$ & 0 \\
\hline 100 & 2 & $2.033 \times 10^{-13}$ & 153.31 & 613.19 & $1.058 \times 10^{-3}$ & & & 933.7 & $1.077 \times 10^{-17}$ \\
\hline 100 & 3 & $9.195 \times 10^{-15}$ & 101.105 & 206.37 & $1.058 \times 10^{-3}$ & & & 314.2 & $4.011 \times 10^{-16}$ \\
\hline 100 & 4 & & 66.866 & 96.728 & $1.058 \times 10^{-3}$ & & & 147.0 & $5.937 \times 10^{-15}$ \\
\hline 100 & 5 & & 46.506 & 54.171 & $1.058 \times 10^{-3}$ & & & 82.48 & $3.989 \times 10^{-14}$ \\
\hline 100 & 6 & & 33.856 & 33.896 & $1.058 \times 10^{-3}$ & & & 51.61 & $1.821 \times 10^{-13}$ \\
\hline 100 & 7 & & 25.580 & 22.877 & $1.058 \times 10^{-3}$ & & & 34.84 & $6.474 \times 10^{-13}$ \\
\hline 100 & 8 & & 19.916 & 16.311 & $1.058 \times 10^{-3}$ & & & 24.84 & $1.925 \times 10^{-12}$ \\
\hline 100 & 9 & & 15.890 & 12.123 & $1.058 \times 10^{-3}$ & & & 18.46 & $5.005 \times 10^{-12}$ \\
\hline 100 & 10 & & 12.938 & 9.3092 & $1.058 \times 10^{-3}$ & & & 14.17 & $6.225 \times 10^{-12}$ \\
\hline
\end{tabular}

\section{LIFETIMES AND LEVEL WIDTHS}

In the previous section the influence of an external electric field on the hydrogen and antihydrogen spectra was considered. It was shown that a significant difference arises for the differential transition probabilities in very weak electric fields. This difference vanishes after integration over photonemission directions, i.e., in the total decay rate. There are also the quadratic in the field summands in Eq. (6) that contribute to the total decay rate. This leads to a strong modification of the corresponding level width. According to the selection rules, the one-photon $n s \rightarrow 1 s$ decay is defined by the emission of a magnetic dipole photon for the isolated atom. However, the admixture of the $n p$ state allows the emission of the electric dipole $E 1$ photon directly to the ground state. In the case when the $2 s$ and $2 p$ states are mixed, this channel gives the main contribution to the level width $[10,11]$. For the critical field strength $D_{c}=475 \mathrm{~V} / \mathrm{cm}$ the width of the $\overline{2 s}$ state is equal to the width of the $2 p$ level. For highly excited states the field strength should be significantly less than $D_{c}$ since the Lamb shift decreases with the increasing of the principal quantum number. Thus a significant shortening of lifetimes for Rydberg states can be expected in a weak electric field.

There are two ways to define level widths: (a) the derivation of the imaginary part of the self-energy correction $[15,16]$ and (b) the summation of all the partial transition probabilities to the low-lying atomic levels. In this section we consider the second procedure. Namely, from Eq. (6) it follows that the additional decay rate due to the electric dipole emission is defined as

$$
\frac{W_{\bar{n} s k s}^{(E 1)}}{e^{2} D_{0}^{2}}=\frac{n^{2}\left(n^{2}-1\right)}{12 \Delta_{1}^{2}} W_{n p k s}^{(E 1)}+\frac{k^{2}\left(k^{2}-1\right)}{36 \Delta_{2}^{2}} W_{n s k p}^{(E 1)} .
$$

Determination of the level width of highly excited states requires consideration of the cascade transitions, which can be described approximately by the one-photon decays to the intermediate atomic levels $[17,18]$. In this case the sum over all the partial transition probabilities $W_{n s k p}^{(1 \gamma)}$ arises for the isolated atom. Therefore, in the presence of the electric field we should write

$$
\Gamma_{\text {tot }}=\Gamma_{n s}+\Gamma_{\overline{n s}}=\sum_{k=1}^{n-1} W_{n s k p}^{(1 \gamma)}+\sum_{k=1}^{n-1} W_{n s k s}^{(1 \gamma)}+\sum_{k=1}^{n-1} W_{\overline{n s} k s}^{(E 1)},
$$

where $\Gamma_{n s}$ is the natural width of the $n s$ level, i.e., the sum of the partial transition probabilities from the initial $n s$ state to all the low-lying $k p$ atomic levels, and $\Gamma_{\overline{n s}}$ represents the contribution of additional decay channels arising in an electric field. Note that the transition probability $W_{n s k s}^{(1 \gamma)}$ corresponds to the $M 1$ decay rate and leads to a negligible contribution to the level width $\Gamma_{\text {tot }}$ (see, for example, Table II).

In principle, the summation over $k$ in Eq. (14) can be performed with the use of the Gordon formula for the transition rates $W_{n s k p}^{1 \gamma}$ and $W_{n p k s}^{(1 \gamma)}$ [19]. However, the dependence on the principal quantum number $k$ should be taken into account as 
TABLE III. The notation is the same as in Table II and the value $D_{c}^{(55)} \approx 3 \times 10^{-5} \mathrm{~V} / \mathrm{cm}$ is used.

\begin{tabular}{|c|c|c|c|c|c|c|}
\hline$n$ & $k$ & $W_{n s k s}^{(1 \gamma)}$ & $W_{n s k p}^{(1 \gamma)}$ & $W_{n p k s}^{(1 \gamma)}$ & $W_{1}$ & $W_{2}$ \\
\hline 55 & 1 & $2.372 \times 10^{-10}$ & & $2.516 \times 10^{4}$ & $1.104 \times 10^{4}$ & \\
\hline 55 & 2 & $1.219 \times 10^{-12}$ & 921.77 & $3.686 \times 10^{3}$ & $1.617 \times 10^{3}$ & $4.09 \times 10^{-13}$ \\
\hline 55 & 3 & $5.499 \times 10^{-14}$ & 608.11 & $1.241 \times 10^{3}$ & 544.3 & $1.52 \times 10^{-11}$ \\
\hline 55 & 4 & & 402.39 & 581.47 & 255.2 & $2.26 \times 10^{-10}$ \\
\hline 55 & 5 & & 280.06 & 325.7 & 142.9 & $1.52 \times 10^{-9}$ \\
\hline 55 & 6 & & 204.06 & 203.86 & 89.46 & $6.93 \times 10^{-9}$ \\
\hline 55 & 7 & & 154.33 & 137.64 & 60.41 & $2.47 \times 10^{-8}$ \\
\hline 55 & 8 & & 120.3 & 98.19 & 43.09 & $7.35 \times 10^{-8}$ \\
\hline 55 & 9 & & 96.111 & 73.028 & 32.05 & $1.91 \times 10^{-7}$ \\
\hline 55 & 10 & & 78.372 & 56.12 & 24.63 & $4.48 \times 10^{-7}$ \\
\hline 55 & 15 & & 35.0001 & 20.657 & 9.066 & $1.16 \times 10^{-5}$ \\
\hline 55 & 30 & & 8.7556 & 4.05 & 1.777 & $2.98 \times 10^{-3}$ \\
\hline 55 & 40 & & 5.2734 & 2.2317 & 0.979 & 0.032 \\
\hline 55 & 45 & & 4.5069 & 1.8316 & 0.804 & 0.089 \\
\hline 55 & 50 & & 4.2245 & 1.6413 & 0.721 & 0.238 \\
\hline 55 & 54 & & 4.4807 & 1.6496 & 0.724 & 0.546 \\
\hline
\end{tabular}

well in the factor $\Delta_{2}$. Furthermore, the applicability of the formula (1) to calculate the transition probabilities requires an analysis of the field strength magnitude $D_{0}$. The perturbation theory is valid when

$$
\left|\frac{\langle n p|e \mathbf{D r}| n s\rangle}{\Delta E_{L}^{(n)}+i \Gamma_{n p} / 2}\right| \lesssim 1
$$

and therefore $|\langle n p|e \mathbf{D r}| n s\rangle| \lesssim \Delta E_{L}^{(n)}$. Using Eq. (2) and the relation $\Delta E_{L}^{(n)} \sim 1 / n^{3}$ [19], we obtain

$$
D_{c}^{(n)} \sim \frac{1}{n^{5}} D_{c},
$$

where $D_{c}$ defines the field strength when a $100 \%$ mixing of the $2 s$ and $2 p$ states in the hydrogen atom occurs, i.e., $D_{c}=$ $475 \mathrm{~V} / \mathrm{cm}$. The field strength scaled as in Eq. (16) is known as the Inglis-Teller limit [20]. Thus the full mixing of $n s$ and $n p$ states should arise in a very weak electric field.

In the following we evaluate the transition rates (13) numerically and give a comparative analysis of the spontaneous and electric-field-induced partial transition probabilities (see Tables II and III). The field strength is evaluated via Eq. (16) for each $n$th initial state. In Table II the contribution of terms quadratic in the field (13) as a function of principal quantum numbers $n$ and $k$ is demonstrated. The one-photon transition probabilities $W_{n s k s}^{(1 \gamma)}, W_{n s k p}^{(1 \gamma)}, W_{n p k s}^{(1 \gamma)}, W_{1} \equiv \frac{n^{2}}{12}\left(n^{2}-\right.$ 1) $e^{2} D_{c}^{(n)^{2}} W_{n p k s}^{(1 \gamma)} / \Delta_{1}^{2}$, and $W_{2} \equiv \frac{k^{2}}{36}\left(k^{2}-1\right) e^{2} D_{c}^{(n)^{2}} W_{n s k p}^{(1 \gamma)} / \Delta_{2}^{2}$ are given in inverse seconds. The calculations of the spontaneous one-photon decay rates can be done, in principle, within the framework of standard quantum electrodynamics and can be found, for example, in [21-23].

In particular, from Table II it follows that the transition probabilities $W_{1}$ and $W_{2}$ arising in the field contribute on the level of partial one-photon decay rates of the $n p$ states. Thus the width of the mixed $\overline{n s}$ state becomes comparable to the natural width $\Gamma_{n p}$ at the very weak field $D_{c}^{(n)}$ and exceeds the natural width of the $n s$ state for the isolated atom. For example, the field strength for the $n=100$ state is estimated as $4 \times 10^{-7} \mathrm{~V} / \mathrm{cm}$ and the corresponding level width $\Gamma \overline{100 s}$ is about $8.5 \times 10^{3} \mathrm{~s}^{-1}$. Therefore, the width of mixed $\overline{100 \mathrm{~s}}$ atomic level is one order larger than the natural width $\Gamma_{100 s}$ and thus the lifetime of the corresponding level is shorter in the presence of the very weak field that can be associated with a stray field.

We considered in more detail the contribution of terms quadratic in the field (13) as a function of the principal quantum number $k$ for the initial state $\overline{55 s}$ at the field strength $D_{c}^{(55)} \approx$ $3 \times 10^{-5} \mathrm{~V} / \mathrm{cm}$. The results listed in Table III show that the mixing of lower states becomes significant for the transitions between nearest atomic levels, whereas the main contribution follows from the first term on the right-hand side of Eq. (13). Summing all the partial transition probabilities in Table III, we obtain $\Gamma_{55 s} \approx 6.5 \times 10^{3} \mathrm{~s}^{-1}, \Gamma_{55 p} \approx 3.2 \times 10^{4} \mathrm{~s}^{-1}$, and the electric-field-induced width $\Gamma_{\overline{55 s}} \approx 1.4 \times 10^{4} \mathrm{~s}^{-1}$.

In principle, the values of Tables II and III should be compared with the level widths evaluated in [24-26]. The main result of the calculations [24-26] is that the values of the corresponding widths can exceed the magnitude of the strongest Lyman- $\alpha$ transition rate $\sim 6 \times 10^{8} \mathrm{~s}^{-1}$ at the fields violating the relation (16) (see, for example, [27]). However, the field strengths considered in our paper correspond rather to very weak fields. Using the electric field of Eq. (16) in the semiempirical expression for the ionization rate $\Gamma_{n n_{1} n_{2} m}$ [28] leads to completely negligible values of the ionization rates.

\section{CONCLUSION}

In this paper we carried out a comparison of the hydrogen and antihydrogen spectra in the presence of an external electric field. It was established that the difference in spectra of the $\mathrm{H}$ and $\overline{\mathrm{H}}$ atoms arises in the presence of a very weak electric field (11). This difference originates from the summands linear in the field in Eq. (6) that have the opposite sign for the hydrogen and antihydrogen atoms. From Eq. (6) it follows that the linear terms vanish in the total transition probability after integration over photon-emission directions that corresponds to the photon detection in all directions simultaneously. 
However, the spectral measurements relate mainly to the detection of the photon emission at a predetermined angle.

A discussion of experiments to detect the emission asymmetry in an electric field for the metastable hydrogen and deuterium atoms can be found, for example, in [29,30]. The main purpose of such experiments is the determination of the Lamb shift. Mohr [12] extended these ideas to high- $Z$ Lamb-shift experiments. The linear (asymmetric) terms give field-strength-dependent contributions of opposite sign to the intensity in the directions parallel and antiparallel to the electric field. In contrast to the hydrogen atom (H-like systems), the contributions of linear terms in Eq. (6) have opposite sign for the antihydrogen atom, i.e., the increase of the relative intensity of radiation for the hydrogen atom should be accompanied by a proportionate decrease of the relative intensity of radiation for the $\overline{\mathrm{H}}$ atom. The recent development of an experiment for the in-flight spectroscopy of antihydrogen atoms [31] provides an opportunity for the corresponding comparison of $\mathrm{H}$ and $\overline{\mathrm{H}}$ spectra in an electric field by the time-of-flight method [32].

It should be stressed that the experiments [6] do not measure the spectrum of antihydrogen atoms but determine the luminescence quenching, estimating in that way the lifetimes of the Rydberg states. As it was reported in [6], the time of antihydrogen trapping is about $1000 \mathrm{~s}$, which is a major step towards precise spectroscopy of the antiatoms [31]. Highresolution comparisons of both systems $(\mathrm{H}$ and $\overline{\mathrm{H}})$ provide sensitive tests of $\mathcal{C P} \mathcal{T}$ symmetry. Any measured difference would point to $\mathcal{C P} \mathcal{T}$ violation [31]. Thus the theoretical study of effects that can mimic such a violation is important. The difference arising in spectra of $\mathrm{H}$ and $\overline{\mathrm{H}}$ atoms in the presence of an external electric field (11) can simulate the effects of the $\mathcal{C} \mathcal{P} \mathcal{T}$-symmetry violation. The difference in spectra (11) depends on the direction of the photon-emission detection: (a) The maximum difference arises when photon emission is detected in the forward or backward side of the field direction $\mathbf{n}_{\mathbf{D}} \mathbf{n}_{\mathbf{k}}= \pm 1$ and (b) the difference vanishes when the photon emission is detected in the direction perpendicular to the field $\mathbf{n}_{\mathbf{D}} \mathbf{n}_{\mathbf{k}}=0$ [Eq. (12)]. The magnitude of the electric-field strength $D_{0}^{\max }$ when this effect has a maximum can be associated with stray fields for Rydberg states (see Table I).

In Table I the values of the maximal difference for the transition rates are illustrated for the field strength (10). The relative difference in spectra $\delta\left(D_{0}^{\max }\right)$ leads to the $10 \%$ discrepancy in spectra of the $\mathrm{H}$ and $\overline{\mathrm{H}}$ atoms. The magnitude of $\delta\left(D_{0}\right)$ was evaluated also for the field $500 \mathrm{~V} / \mathrm{m}$, which we associate with the experimental value. There are two competing contributions leading to the same effect: (i) mixing of the initial $n s$ and $n p$ states and (ii) mixing of the final $k s$ and $k p$ states. Mixing of lower atomic levels should lead to more significant violation in the spectra of hydrogen and antihydrogen atoms in the case of stronger fields (10). We should note that the formal $T$ noninvariance of the scalar product $\mathbf{n}_{\mathbf{D}} \mathbf{n}_{\mathbf{k}}$ is compensated by the dependence on $\Gamma_{n p}$ (see [14]).

In view of experiments [6], using the example of $n s$ states, we have evaluated the partial decay rates for Rydberg states of the hydrogen atom in the presence of an external electric field. The calculation of the total transition probabilities for the different decay channels of highly excited states allows the definition of corresponding level widths as a sum over all the partial decay rates. The Rydberg state has a long lifetime in view of the cubic decrease of the corresponding transition probabilities [19] with respect to the principal quantum number of the initial state. The most interesting conclusion following from our estimates is that even in the presence of the very weak homogeneous electric field, the states $n s$ and $n p$ become indistinguishable in spectroscopic experiments (Table II). Primarily this occurs because while the $n s \rightarrow 1 s+1 \gamma(E 1)$ decay is forbidden by the selection rules, emission of an electric dipole photon is allowed in the presence of an external electric field. The additional $E 1$ decay channels to the ground and intermediate atomic levels are described by the terms quadratic in the field (13) and are comparable to the partial decay rates of the $n p \rightarrow k s+1 \gamma(E 1)$ or $n s \rightarrow k p+1 \gamma(E 1)$ transitions for the isolated atom (Table II). For example, the $100 \%$ mixing of $100 \mathrm{~s}$ and $100 \mathrm{p}$ states occurs at the field of the order of $4 \times 10^{-7} \mathrm{~V} / \mathrm{cm}$, making the decays of these states identical. The presence of an external electric field should be taken into account for both initial and final (intermediate) states. The detailed study of mixing of the lower-lying states can be done via the results of Table III. In particular, from Table III it follows that the mixing of low-lying states becomes important for the transitions between neighboring levels. Thus the stray fields lead to a substantial change of level widths.

We should note that even very weak fields can lead to significant changes in the emission spectra of hydrogen and antihydrogen atoms in Rydberg states. First, the maximal asymmetry (identical for any $n s$ states) in the hydrogen and antihydrogen spectra arises in fields of the order of $D_{0}^{\max }$ (see Table I). Second, the results of Tables II and III show that the $100 \%$ mixing of $n s-n p$ states occurring in the field of the order of $D_{c}$ substantially increases the depopulation Rydberg $n s$ states. The magnitudes of the field strengths $D_{0}^{\max }$ and $D_{c}$ were calculated for each $n s$ state to assess the maximal contribution of both effects. It is shown that electric fields $D_{0}^{\max }$ and $D_{c}$ leading to the asymmetry and $100 \%$ mixing of Rydberg $n s-n p$ states can vary by 13 orders of magnitude. Thus the results impose significant restrictions on the experimental settings. The efficient control of external electric fields or the photon-emission angles is required. The counting of the photon emission in the direction perpendicular to the field would reduce to zero the asymmetry of the $\mathrm{H}$ and $\overline{\mathrm{H}}$ spectra and control of the field strength can minimize the line broadening.

\section{ACKNOWLEDGMENTS}

The authors are grateful to Professor L. N. Labzowsky for helpful discussions. D.S. acknowledge support from the Russian Foundation for Basic Research through Grants No. 11-02-00168-a and No. 14-02-20188. The authors also acknowledge support from Saint-Petersburg State University through Grant No. 11.38.227.2014.
[1] A. Huber, B. Gross, M. Weitz, and T. W. Hänsch, Phys. Rev. A 59, 1844 (1999).
[2] M. Niering, R. Holzwarth, J. Reichert, P. Pokasov, Th. Udem, M. Weitz, T. W. Hänsch, P. Lemonde, G. Santarelli, M. Abgrall, 
P. Laurent, C. Salomon, and A. Clairon, Phys. Rev. Lett. 84, 5496 (2000).

[3] C. G. Parthey, A. Matveev, J. Alnis, B. Bernhardt, A. Beyer, R. Holzwarth, A. Maistrou, R. Pohl, K. Predehl, T. Udem, T. Wilken, N. Kolachevsky, M. Abgrall, D. Rovera, C. Salomon, P. Laurent, and T. W. Hänsch, Phys. Rev. Lett. 107, 203001 (2011).

[4] D. Arnoult. F. Nez, L. Julien, and F. Biraben, Eur. Phys. J. D 60, 243 (2010).

[5] E. Peters, D. C. Yost, A. Matveev, T. W. Hänsch, and T. Udem, Ann. Phys. (Berlin) 525, L29 (2013).

[6] The ALPHA Collaboration, Nat. Phys. 7, 558 (2011).

[7] R. Bluhm, V. A. Kostelecky, and N. Russell, Phys. Rev. Lett. 82, 2254 (1999).

[8] L. Labzowsky and D. Solovyev, Phys. Rev. A 68, 014501 (2003).

[9] D. A. Solov'ev, V. F. Sharipov, L. N. Labzovskii, and G. Plunien, Opt. Spectrosc. 104, 509 (2008).

[10] D. Solovyev, V. Sharipov, L. Labzowsky, and G. Plunien, J. Phys. B 43, 074005 (2010).

[11] Ya. I. Azimov, A. A. Anselm, A. N. Moskalev, and R. M. Ryndin, Zh. Eksp. Teor. Fiz. 67, 17 (1974) [Sov. Phys. JETP 40, 8 (1975)].

[12] P. J. Mohr, Phys. Rev. Lett. 40, 854 (1978).

[13] D. Solovyev, L. Labzowsky, A. Volotka, and G. Plunien, Eur. Phys. J. D 61, 297 (2011).

[14] Ya. B. Zel'dovich, Zh. Eksp. Teor. Fiz. 39, 1483 (1960) [Sov. Phys. JETP 12, 1030 (1961)].

[15] O. Yu. Andreev, L. N. Labzowsky, G. Plunien, and D. A. Solovyev, Phys. Rep. 455, 135 (2008).
[16] T. Zalialiutdinov, D. Solovyev, L. Labzowsky, and G. Plunien, Phys. Rev. A 89, 052502 (2014).

[17] L. Labzowsky, D. Solovyev, and G. Plunien, Phys. Rev. A 80, 062514 (2009).

[18] T. Zalialiutdinov, Yu. Baukina, D. Solovyev and L. Labzowsky, J. Phys. B 47, 115007 (2014).

[19] H. A. Bethe and E. E. Salpeter, Quantum Mechanics of Oneand Two-Electron Atoms (Springer, Berlin, 1957).

[20] T. F. Gallagher, Rydberg Atoms (Cambridge University Press, Cambridge, 1994).

[21] O. Jitrik and C. F. Bunge, J. Phys. Chem. Ref. Data 33, 1059 (2004).

[22] W. L. Wiese and J. R. Fuhr, J. Phys. Chem. Ref. Data 38, 565 (2009).

[23] A. M. Puchkov and L. N. Labzovskii, Opt. Spectrosc. 106, 153 (2009).

[24] A. Alijah, J. T. Borad, and J. Hinze, J. Phys. B 19, 2617 (1986).

[25] I. D. Feranchuk and L. X. Hai, Phys. Lett. A 137, 385 (1989).

[26] L. Fernández-Minchero and H. P. Summers, Phys. Rev. A 88, 022509 (2013).

[27] V. V. Kolosov, J. Phys. B: At. Mol. Phys. 20, 2359 (1987).

[28] R. J. Damburg and V. V. Kolosov, J. Phys. B 12, 2637 (1979).

[29] G. W. F. Drake, P. S. Farago, and A. van Wijngaarden, Phys. Rev. A 11, 1621 (1975).

[30] A. van Wijngaarden and G. W. F. Drake, Phys. Rev. A 17, 1366 (1978).

[31] N. Kuroda et al., Nat. Commun. 5, 3089 (2014).

[32] H. Gould and R. Marrus, Bull. Am. Phys. Soc. 22, 1315 (1977). 